\title{
The Research on the Connotation and Structure of Chinese College Teachers' Psychological Capital
}

\author{
Wang Bin and Lanzhen Zhu
}

\begin{abstract}
This paper studies the connotation and structure of Chinese college teachers' psychological capital by means of literature deductive, interview and questionnaire. The results show that the connotation of Chinese college teachers' psychological capital refers to their psychological elements and abilities that meet the standard of positive organization behavior, promote job performance and belong to the state-like. Besides, those elements are able to be developed and measured. Its structural dimension belongs to the second-order two-factor and eight dimensions. During eight dimensions interpersonal psychological capital includes four dimensions, tolerance, respect, modesty and dedication. Transactional psychological capital also includes four dimensions, confidence, hope, enterprise and resiliency. Through reliability test, the $\alpha$ coefficients of eight dimension are more than 0.7. Among them the $\alpha$ coefficient of interpersonal psychological capital is 0.842 , transactional psychological capital 0.888 , integral structure 0.955 and content validity inspection value 8.96 which shows the good structure and content validity. The study not only establishes the foundation for the research on the measurement, features and influencing factors of college teachers' psychological capital and enriches the theory of psychological capital, but also provides theoretical basis and reference for human resource management and sustainable development of college physical education teachers.
\end{abstract}

Index Terms-Psychological capital, HRM (human resource management), connotation; structure.

\section{INTRODUCTION}

In recent years, with the rapid development of China's higher education reform, how to seek for a positive new model that can effectively tap individual potential and improve individual subjective initiative and school-running efficiency under the existing human resource management model has become an urgent problem to be solved in Chinese universities' human resource management. Fred Luthans, the founder of American organizational behavior management, with his team put forward the concept and theory of psychological capital in 2004. They believed that psychological capital is beneficial for organizations and individuals to obtain strategic resources and enhance their advantages of sustainable competition like human capital and social capital. [1] Psychological capital is a developed, measurable and positive psychological resource that can effectively promote personal development and improve efficiency. [2] Psychological capital has been widely used in

Manuscript received October 5, 2019; revised April 2, 2020.

Wang Bin is with Sport and Health Science College of Zhejiang Normal University, Jinhua, Zhejiang, 321004, China (e-mail: wangbin@ zjnu.cn).

Lanzhen Zhu is with Yiwu Industrial and Commercial College, Yiwu, 321004, China (corresponding author; email:403534545@qq.com). many disciplines and fields since it was proposed. The content of the study involves the connotation of psychological capital, measurement, antecedent and consequent variables, mediator, moderator and intervention management. The research objects include enterprise staff, primary and secondary school teachers, students, nurses, civil servants, etc [3]. After surveying the existing literature, the study on the connotation and structure of psychological capital of college teachers hasn't been found so far. Taking the psychological capital of college teachers as the study object and using ways of literature deductive, interview and so on on the basis of making full use of previous research results, this study is to explore the connotation and structure of college teachers' psychological capital for establishing the foundation of the study of characteristics of college teachers' psychological capital and long-term intervention, enriching the psychological capital theory and injecting fresh blood into the human resource management and the sustainable development of college teachers.

\section{The Connotation of Psychological CAPITAL of CHINESE COLLEGE TEACHERS}

\section{A. Overview of Psychological Capital Connotation}

Influenced by positive psychology, professor Fred Luthans, the former chairman of management in the United States, and his collaborators have brought the ideas of positive psychology into the field of talent management and organizational behavior creatively and smartly and put forward the concept of "psychological capital". Initially, Luthans with his research members defined psychological capital as that positive and essential psychological elements of individuals conform to the psychological state of the standard of positive organizational behavior (POB) and it enables individuals to obtain competition advantages through targeted investment and development [4]. Its core essence consists of four aspects: 1) It is based on the positive psychology; 2) It meets the five criteria of positive organizational behavior (POB) (e.g. theory and study foundation, novelty and specialty in the field of organizational behavior, effective measure, state and positive influence on job performance); 3) A transcendence of human and social capital. Psychological capital emphasizes the individual, which is more concerned about who you are [5]. In 2007 Luthans and others revised its definition as that psychological capital is a kind of positive psychological state of individuals in the process of growth and development. It also includes four aspects: 1) The state of making efforts and having confidence to achieve success when faced with challenging work; 2) Positive attributions (optimism) for 
present and future goals; 3) Great hope to achieve goals and the adjustment of approaches to achieve goals when it is necessary for achieving success; 4) Be persistent and tenacious to achieve success when faced with adversity and problems. [6] At the same time, Fred -Luthans believed that the difference between psychological capital and general positive psychology lies in that the former belongs to the variable of state-like. It is exactly the characteristic of state-like that makes psychological capital not become erratic, emotional and too stubborn to change, which establish a foundation for developing the value of psychological capital in the future. Therefore, psychological capital is a collection including people's positive psychological abilities and it can be developed, measured and managed effectively through the intervention of each components. In addition, it is relatively stable over a period of time [7]. The research about positive psychology shows that there are both negative and positive forces at the bottom of everyone's heart. The negative aspects of human nature can be eliminated or suppressed and an individual can become a self-actualized person when the inherent positive forces of human beings are cultivated and developed.

\section{B. Research Status of Psychological Capital Connotation}

At present, most scholars have the same understanding of the connotation of psychological capital. When defining the concept of single-group psychological capital, it is mainly on the basis of Fred-Luthans's definition of the connotation of psychological capital, that is on the basis of following the criteria of $\mathrm{POB}$ to derive the definition of single-group psychological capital. For instance, when the scholar, KeJianglin with others conducted localization research on the psychological capital theory, they defined psychological capital as individuals' measurable and exploitable positive attitude or psychological ability promoting work performance in the process of dealing with others under the Chinese organizational context. [8] When developing psychological capital questionnaire for primary and secondary school teachers, Zhang Wen (2010) believed that the operational definition of teachers' psychological capital is the positive psychological state in the teaching process. Its features are as followings: 1) Be fully confident when successfully completing challenging teaching tasks and make necessary efforts for the teaching task; 2) Hold the persistent attitude towards the realization of teaching objectives and be able to re-choose the route to achieve the objectives when necessary; 3) Hold the optimistic and positive attitude towards current and future teaching work (optimism); 4) Be able to bear pressure when encountering teaching difficulties, recover quickly and adopt various effective methods to achieve success in teaching tasks (resilience). In other words, the psychological capital of primary and secondary school teachers includes four dimensions: confidence, hope, optimism and resilience [9]. Wu Weijiong (2011) defined the psychological capital of primary and secondary school teachers as a collaborative collection of psychological abilities that meet the criteria of psychological capital evaluation in the teaching process, which plays a positive role in promoting their work performance. Jiang Suqin (2010) defined psychological capital of college students as a positive psychological factor with exploitable, measurable and stable characteristics that can lead to individuals' positive behaviors [10].

\section{The Connotation of Psychological Capital of Chinese College Teachers}

Psychological capital of Chinese university teachers is a sub-concept of psychological capital, which has common parts compared with psychological capital of different groups and backgrounds. Nevertheless, due to individuals' collection of psychological resources can be effected by the differences on culture, social division of labor, psychological characteristics and social experience, these subjective and objective factors will inevitably lead to the fact that the psychological capital of Chinese college teachers also has its unique connotation and structure.

Therefore, our study synthesizes predecessors' research results, uses study methods of literature deductive, interview and questionnaire and combines with the working characteristics of college teachers and college teachers' work rules and the content of college teachers' work regulations and Teachers' Law. We define the connotation of psychological capital of Chinese college teachers as the measurable, exploitable and positive psychological elements promoting the work performance which are shown in the work situation. This kind of psychological elements mainly include eight aspects as followings: 1) Enterprise: Challenge oneself in work, pursue higher goals and desire to achieve success; 2) Confidence: The strong competency in work and the encourage to dare to show own talent; 3) Hope: Hold the optimistic attitude to adversity without depression and desperation; 4) Tenacity: $\mathrm{Be}$ able to endure adverse conditions, keep calm and try to change the status quo when facing difficulties or crisis in work; 5) Respect: Show respect to others and attach importance on etiquette to let others have the feel of priority; 6) Modesty: Evaluate oneself and others objectively and keep one's promise; 7) Gratitude: Be grateful, put the interests of schools and students first and be ready to devote without asking for return; 8) Accept different people who have different character and different cultivation and tolerant others' shortcomings. These eight psychological elements fully reflect the diversity and complexity of the connotation of psychological capital of university teachers.

\section{The Structure of Psychological Capital of Physical Education Teachers in COLleges}

\section{A. Research Status of Psychological Capital Structure}

Due to the differences in understanding of the concept of psychological capital, the research results on the structural dimension of psychological capital show the character of diversification. The representative literature is summarizedas followings: 1) The view of two-dimension. Scholars such as Goldsmith (1997) holding this view believe that the structural dimension of psychological capital mainly includes two aspects, control point and self-esteem. 2) The view of three-dimension. Scholars such as Avey (2006) holding this view believe that psychological capital is a core concept of higher level formed by the combination of hope, optimism and resiliency. [11] 3) The view of four-dimension. Scholars 
such as Luthans holding this view believe that the structure of psychological capital is mainly composed of self-efficacy, hope, optimism and resiliency. In addition, there are a few scholars like Judge (2001) and Cole (2006) who consider the structure of psychological capital is composed of self-esteem, self-efficacy, control points and emotional stability. 4) The view of multiple dimensions. Seligman held the belief that as long as the dimensions belong to the positive psychological condition or psychological elements, they should be divided into the structural dimension of psychological capital. Therefore, the structural dimension of psychological capital should be open. Fred $\bullet$ Luthans in later studies also deemed that keeping the dynamic character of the structure of psychological capital is not only necessary but also in line with the objective law of the development of concepts andessence of things. As long as other positive psychological elements have solid theoretical basis and can be measured and developed, they can also be divided into the structure of psychological capital [11]. Luthans' viewpoint has become a solid theory of the emergence and development of multiple dimensions of psychological capital structure. According to Luthans' theory, many scholars put forward many viewpoints about multiple dimensions of psychological capital structure through research, for example, Letcher's (2004) five dimensions including extroversion, openness, emotional stability, agreeableness and responsibility. In addition, on the basis of Jensen's four dimensions Page (2004) added the dimension of integrity and Chinese scholar, Cao Mingqiraised up his viewpoint that (2006) structural dimensions consist of six aspects of hope, optimism, subjective well-being, emotional intelligence, resiliency and citizenship's organizational behavior.

\section{B. Psychological Capital Structure Theory of Fred $\bullet$ Luthans}

Through the analysis and screening of human's state-like, Fred-Luthansraised up that the core structure of psychological structure is composed of hope, self-efficacy, optimism, resiliency and so on [12]. Hope, American scholar, Rick Snydr (2002) defined it as a positive motivational state formed by the experience generated by individuals' interaction between motivation of success and approaches of success [11]. Through study he confirmed that hope is a state of cognition or thinking, in which individuals can set realistic but challenging task objectives and achieve them through the determination of self-direction, energy and perception of internal control. Individuals with high hope can choose more reasonable ways that people are willing to find it and have the ability to find it to achieve goals [13]. Self-efficacy, also known as confidence, was first put forward by the famous psychologist, Bandura. He defined it as the belief that in a specific situation individuals stimulate motivation and mobilize cognitive resources to complete tasks. On the basis of social cognition Bandura further elaborated that self-efficacy is built on the cognitive processes of advance thinking, observation, symbolization, self-regulation and self-reflection [13]. He thought that self-efficacy is related to specific fields. Specifically, it is high in individuals' familiar fields and vice versa. Self-efficacy is state-like and variable because it will be influenced by people who are important for them, is based on the connection or proficiency of tasks and can be improved in many ways. Luthans also considered that self-efficacy is not only embodied in individuals (individual efficacy), but also exists in collective organizations (collective efficacy) and both individual efficacy and collective efficacy are positively correlated with individual or team's performance [13]. Optimism: Optimists tend to make unstable, specific and external attributions to negative events and believe that as long as they make efforts, they will embrace success on the basis of overcoming and getting rid of difficulties. On the contrary, pessimists usually think that failure is on account of their own fault and success is because of their good luck [13]. Resiliency: In clinical psychology Ann S. Masten and Reed defined resilience as a psychological phenomenon that can adopt a positive attitude to adapt difficult or dangerous situations. Through theoretical analysis and experimental research, Luthans et al concluded that resiliency refers to the daily psychological skills and advantages of individuals who are at any age and have any mental condition, which can be found, measured, maintained and developed. In addition, resiliency is an exploitable psychological element that enables individuals to quickly recover from adversity and even positive events and surpass it and it is of great significance to improve individuals abilities [13]. The four core structural elements above are not in an additive way, but in a synergistic way to exert influences. The overall effect of psychological capital can be greater than the sum of its elements. For instance, a person with hope in his heart must have his path and motivation to accomplish his goals and his power to overcome difficulty will be stronger. Therefore, there will be more resiliency to accomplish goals. That is, confident people are bound to apply hope, optimism and resiliency to specific tasks in a particular area of their work and life. A resilient person is better at exploiting the adaptive mechanisms necessary for realistic and flexible optimism. The confidence, hope and resiliency of psychological capital can contribute to form an optimistic and explanatory style by understanding the internalized perception as something oneself can control [14].

TABLE I: THE SELECTED COGNITIVE AdVANTAGES, EMOTIONAL AdVANTAGES, Social AdVANTAGES AND Higher LEVELS' ADVANTAGES

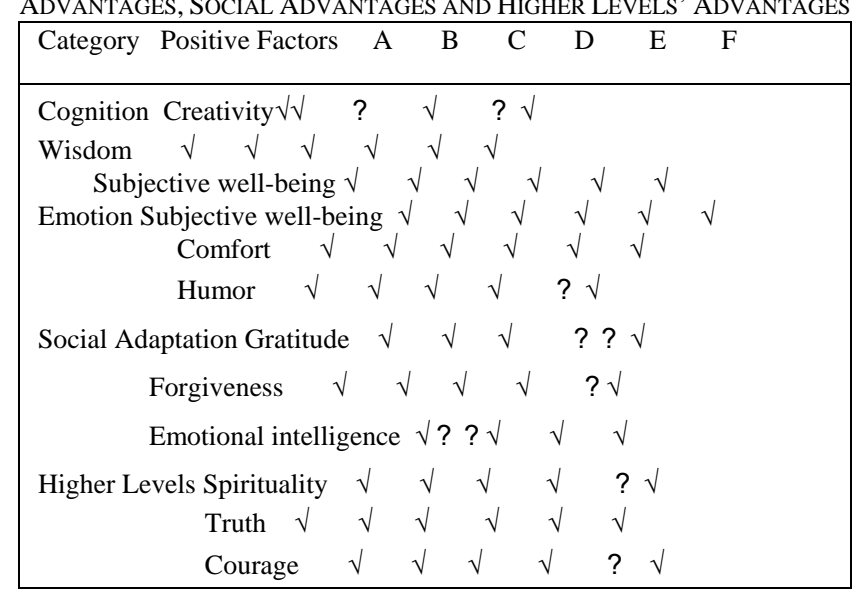

Note: A indicates whether there is a theoretical basis; B indicates whether individual traits are relatively fixed; $\mathrm{C}$ indicates whether the characteristic of state-like can be developed; $\mathrm{D}$ indicates whether it is measurable; $\mathrm{E}$ indicates whether it is related with job performance; $F$ indicates whether it's related to other positive outcomes. [14] 


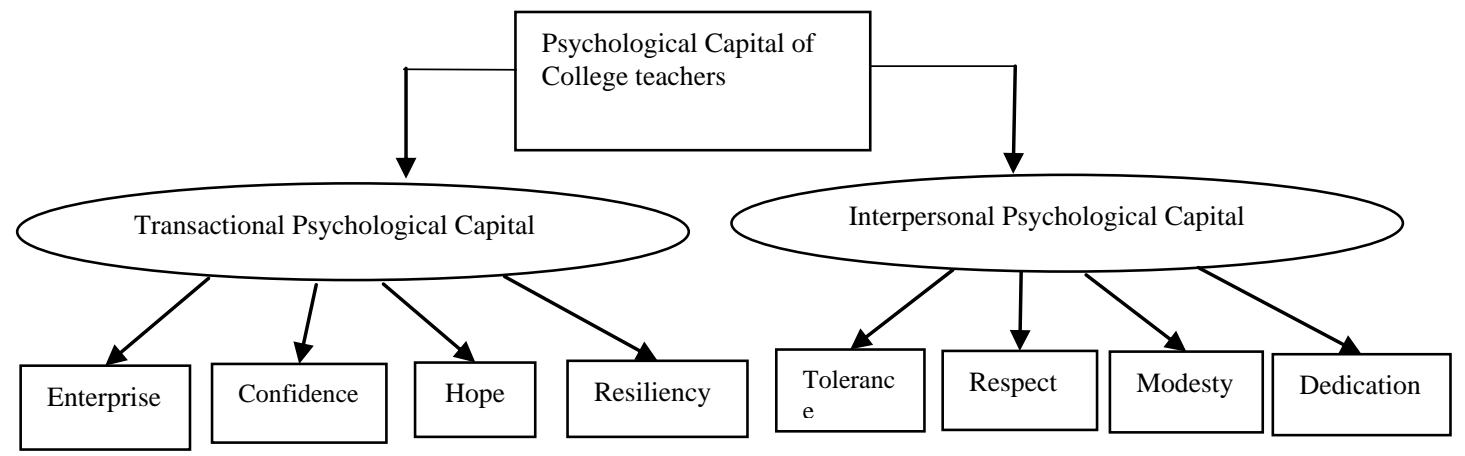

Fig. 1. Psychological capital structure of college teachers.

TABLE II: THE STRUCTURAL Dimension AND OPERATIONAL DEFINITION OF PSYCHOLOGICAL CAPITAL OF COLLEGE TEACHERS

\begin{tabular}{|c|c|c|c|}
\hline \multicolumn{2}{|c|}{ Transactional Psychological Capital } & \multicolumn{2}{|c|}{ Interpersonal Psychological Capital } \\
\hline Confidence & $\begin{array}{l}\text { Believe in personal abilities and have the } \\
\text { courage to show talents in different ways on } \\
\text { different occasions }\end{array}$ & Tolerance & $\begin{array}{l}\text { Be able to understand and tolerant others' } \\
\text { deficiencies and faults; be able to be tolerant and } \\
\text { accept others. }\end{array}$ \\
\hline Hope & $\begin{array}{l}\text { Hold positive and optimistic attitude towards } \\
\text { uncertain things; make efforts to solve adverse } \\
\text { things and actively work to solve and be not } \\
\text { easy tobe in depression and despair. }\end{array}$ & Respect & $\begin{array}{l}\text { Respect others' social attributes and do not } \\
\text { belittle others' talents;be careful about personal } \\
\text { etiquette to give others a sense of priority }\end{array}$ \\
\hline Enterprise & $\begin{array}{l}\text { Have high personal ambition and pursuit high } \\
\text { goals; enjoy challenges and be eager to achieve } \\
\text { success }\end{array}$ & Modesty & $\begin{array}{l}\text { Be able to objectively know personal weaknesses } \\
\text { and others' strengths; learn from others: and keep } \\
\text { promises. }\end{array}$ \\
\hline Resiliency & $\begin{array}{l}\text { When encountering difficulties or crisis be calm } \\
\text { to face adversity and do not give up easily; hold } \\
\text { perseverance and persistence and try to change } \\
\text { the adverse situation }\end{array}$ & Dedication & $\begin{array}{l}\text { Be grateful and be willing to dedicate oneself; be } \\
\text { willing to help others and think for the team. }\end{array}$ \\
\hline
\end{tabular}

Fred $\bullet$ Luthans et al pointed out in later researches that with the constant improvement and development of positive psychology knowledge system, not only these four abilities meet the standard of POB, many other positive factors also have a solid theoretical foundation, strong application and can be measured and developed. Consequently they will also be structural elements of the psychological capital [11]. At the same time, Fred $\bullet$ Luthans and his team of researchers discussed some other positive psychological capacities from four aspects, cognitive advantages, emotional advantages, social advantages and higher levels' advantages. The results are shown in Table I.

The above theories and research methods exploit thinking and establish a foundation for the study of psychological capital theory's subsequent development and the research of cross-cultural. At the same time, they offer help for us to study college teachers' concept and structure of psychological capital.

\section{The Structure of Psychological Capital of College Teachers}

Psychological capital of college teachers is a subtype of psychological capital. Nevertheless, cultural differences, social division of labor, personal psychological characteristics, social experience and so on will affect the collection of resources in people's life, which inevitably lead to that psychological capital of college teachers also has its unique content structure. Since ancient times, China has been a country that attaches great importance to human relations and once there were many humanist thinkers emerging out who were leading the world. College is a place with rich humanistic atmosphere and complex interpersonal relations while college physical education teachers are also a group with distinct personality, complex professional categories, abundant experience and major background. The quality of interpersonal relationship will have a great impact on the improvement of job performance and professional success and at the same time, others' identification of individual attitude and behavior relies on the establishment of interpersonal relationship. The positive attitude that individuals need to have when dealing with interpersonal relationship effectively is bound to be closely related to the standard or culture that are widely accepted by the society. Therefore in this context, it is necessary to consider college teachers' measurable and exploitable positive psychological elements promoting job performance from the aspect of conducting oneself.

This study is based on theFred $\bullet$ Luthans's theories and methods, synthesizes previous research results, defines the connotation and content of psychological capital of college teachers for us and defines the structure of psychological capital of college teachers based on the research methods of literature deductive and interview. It considers that psychological capital of college teachers belongs to the structure of second-order two-factor and eight dimensions. Namely, interpersonal psychological capital contains tolerance, respect, modesty and dedication. Transactional psychological capital contains enterprise, confidence, hope and resiliency. As is shown in Figure 1:

The structural elements are defined as follows (see Table II).

From the structure and definition of psychological capital of college teachers, we can see that the psychological capital structure shows its multiple dimensions inevitably because college teachers in China are effected by the influence of cultural environment, work nature and other factors.

D. The Examination of Psychological Capital Structure of College Teachers 


\section{1) Reliability test of psychological capital structure of} college teachers

Reliability is the dependability and stability between variables. The commonly used methods to test reliability are test-retest reliability, rater reliability, coefficient of internal consistency, alternate-form reliability and so on. Among those methods L. J. Cronbach's $\alpha$ coefficient' coefficient of internal consistency is the commonest one. The formula is:

$$
\alpha=\frac{K}{K-1}\left(1-\frac{\sum S_{i}^{2}}{S^{2}}\right)
$$

$K$ is the total number of questions in the scale $\sum S_{i \text { is the }}^{2}$ sum of variance of scale items; $S 2$ is the variance of the sum of items in the scale. $\alpha$ coefficient's threshold is between 0 and 1 . The values to judge are shown in Table III. [15]

TABLE III: THE PRINCIPLE OF JUDGEMENT OF THE INDEX OF COEFFICIENT OF INTERNAL CONSISTENCY

\begin{tabular}{|l|l|l|}
\hline $\begin{array}{l}\text { Coefficients of Internal Consistency } \\
\text { Reliability }\end{array}$ & Levels / Construct & The Whole Scale \\
\hline$\alpha$ coefficient $<0.05$ & Not ideal and give up & Extremely not ideal and give up \\
\hline $0.05 \leq \alpha$ coefficient $<0.06$ & Acceptable; revise & Not ideal; reprogram or revise \\
\hline $0.06 \leq \alpha$ coefficient $<0.07$ & Not very good & Reluctantly accept; revise appropriately \\
\hline $0.07 \leq \alpha$ coefficient $<0.08$ & Good (highreliability) Acceptable & \\
\hline $0.08 \leq \alpha$ coefficient $<0.09$ & Ideal (Very high reliability) Good & Very ideal \\
\hline$\alpha$ coefficient $\geq 0.09$ & Very ideal; Very high reliability & \\
\hline
\end{tabular}

TABLE IV: Psychological CAPITAL StRucture of PhySiCAl EduCATION TEACHERs In COLLEGES CRON BACH A COEFFICIENT TABLE

\begin{tabular}{|c|c|c|c|c|c|c|c|c|c|}
\hline Dimension & Confidence & Hope & Enterprise & Resiliency & Tolerance & Respect & Modesty & Dedication & Total \\
\hline$\alpha$ coefficient & .798 & .780 & .762 & .701 & .803 & .752 & .765 & .722 & .933 \\
\hline
\end{tabular}

Table IV shows that each dimension's $\alpha$ coefficients are more than 0.70 . Among them the value of dimension of respect is more than 0.803 . The coefficient of transactional psychological capital is 0.840 ; the coefficient of interpersonal psychological capital is 0.868 and the $\alpha$ coefficient of overall structure is 0.933 . As we can be seen from table 4, Psychological Capital Structure of Physical Education Teachers in Colleges shows it good reliability.

\section{2) Validity test of psychological capital structure of college teachers}

Validity refers to being able to test the extent of that psychological elements can be achieved which this test wants to measure. Validity is goal-oriented so the value of validity test is only the value of its special purpose without generalized deduction. Validity generally includes content validity, criterion-related validity and construct validity [15]. The study is mainly aimed at surveying the construct validity of psychological capital's structure. There are 23 teachers participating in the questionnaire survey, including 10 persons holding managing work, 16 professors, 6 associate professors, 6 psychology experts, and 12 persons with doctorate. The contents of the evaluation include the instruction, items, arrangement, the structure of psychological capital and so on. They have close contacts and communication with members of the research group in the academic circle. The results show that the validity of psychological capital structure of college teachers is 8.94 which fully indicates that the content validity of the structure is good.

\section{CONCLUSION}

Psychologists once said that psychological capital is the re-cognition of traditional human resources and strong psychological capital is the guarantee of the unlimited development of human potential. Management experts think that psychological capital has found a new way for people who study psychologist to compete in this age and that is a deep insight and new experience of human resource management research. We must fully recognize and explore the psychological capital on the basis of the support of social environment and culture and individuals' development and success. The second-order two-factor and eight dimensions of positive psychological elements which can promote job performance and can be measured and developed in the work of college teachers, are the main body of their structure and content in theory or in practice and express the connotation and structure of college teachers' psychological capital completely and accurately. In the future, it will play a theoretical and practical role in the sustainable development of human resource management in colleges. Just as Fred•Luthans said, psychological capital is the inexhaustible power stored in the bottom of our hearts and the source of sustainable development of life.

\section{CONFLICT OF INTEREST}

The authors declare no conflict of interest.

\section{AUTHOR CONTRIBUTIONS}

Wang Bin conducted the research and analyzed the data and wrote the paper. Lanzhen Zhu was the corresponding author, responsible for the translation of English materials, data statistics and paper discussion; both authors had approved the final version.

\section{REFERENCES}

[1] F. Wang, "University librarians' psychological capital: Definition, measurement and validity," Journal of Library Science in China.

[2] Fred•Luthans[America], Psychological Capital, Beijing: China Light Industry Press, 2008, p. 17;

[3] B. Wang, "The origin, status and development of psychological capital," in Proc. Sports History and Culture High-end Forum, 2018.

[4] FredLuthans[America], Psychological Capital, Beijing: China Light Industry Press, 2008, p. 15.

[5] FredLuthans[America], Psychological Capital, Beijing: China Light Industry Press, 2008, p. 18. 
[6] Y. Xu, "The preliminary study on psychological capital in human resource management," Guide Business, 2009.

[7] B. M. Shi, "The effect of proactive personality on college students' creativity: The mediation role of psychological capital and creative motivation," Zhejiang University, 2014.

[8] J. L. Ke, "Psychological: The development of local scale and its comparison of China and West," Acta Psychologica Sinica, 2008

[9] W. Zhang, "The compilation and characteristic analysis of psychological capital questionnaire for primary and secondary school teachers," Southwest University, 2010.

[10] F. Wang, Journal of Library Science in China.

[11] B. Wang, "Review on psychological capital research and its enlightenment to the study of college physical education teachers' psychological capital," Bulletin of Sport Science \& Technology, 2019.

[12] M. Li, "The construction and related research of psychological capital of college teachers," Henan University, 2010.

[13] Fred•Luthans[America], Psychological Capital, Beijing: China Light Industry Press, 2008, p. 19.

[14] B. Wang, "The origin, status and development of psychological capital," in Proc. Sports History and Culture High-end Forum, 2018.

[15] In 1974 American Psychological Association published Standards for Educational and Psychological Tests.

Copyright $\odot 2020$ by the authors. This is an open access article distributed under the Creative Commons Attribution License which permits unrestricted use, distribution, and reproduction in any medium, provided the original work is properly cited (CC BY 4.0).

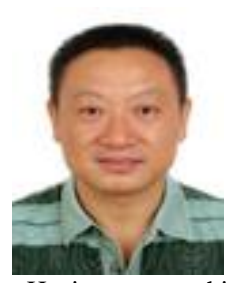

Wang Bin was born in Tianshui city, Gansu province, 1964.07. His educational background: Physical Education Institute of Northwest Normal University, Physical Education, Bachelor of Science, 1985.07. Shanghai Institute of Physical Education, Humane Sociology, Master of Science, 2002.07.China Research direction: sports, human sociology, physical education and management.

He is now teaching in Zhejiang Normal University School of Physical Education and Health Science, professor, masterundefineds degree supervisor. The main monographs published are:

[1]. Folk sports in ancient China. Beijing China Social Science Press. 2013.05.

[2].A study on the characteristics and influencing factors of psychological capital of physical education teachers in colleges and universities in China. Tianjin. Tianjin Academy of Social Sciences Press. 2019.05.

Professor Wang Bin, a member of the Committee of Chinese Sports and Cultural Experts, the director of the Chinese Institute of Sports History and vice-chairman of Zhejiang Institute of Physical Education.

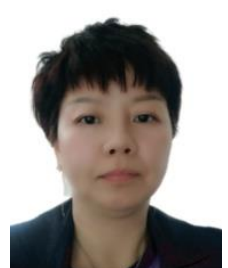

Lanzhen Zhu is the corresponding author and is now working as an associate professor at Yiwu Industria and Commercial College. She holds a M.A. in administrative management from Shanghai Normal University, (China, 2009). Previously she has worked as a lecturer at the Sichuan International Studies University (SISU, China). Research interests: College teachers' sustainable development, sociology of culture and cultural studies. 